\title{
Cognitive behavioural therapy for insomnia reduces sleep apnoea severity: a randomised controlled trial
}

\author{
Alexander Sweetman (10 ${ }^{1}$, Leon Lack ${ }^{1,2}$, R. Doug McEvoy ${ }^{1,3}$, Nick A. Antic ${ }^{1,3, \dagger}$, \\ Simon Smith ${ }^{4}$, Ching Li Chai-Coetzer ${ }^{1,3}$, James Douglas ${ }^{5}$, Amanda O'Grady ${ }^{1}$, \\ Nicola Dunn ${ }^{5}$, Jan Robinson ${ }^{5}$, Denzil Paul ${ }^{1}$, Danny Eckert (10 ${ }^{1}$ and \\ Peter G. Catcheside ${ }^{1}$
}

Affiliations: ${ }^{1}$ The Adelaide Institute for Sleep Health: a Centre of Research Excellence, College of Medicine and Public Health, Flinders University, Adelaide, Australia. ${ }^{2}$ The Adelaide Institute for Sleep Health: a Centre of Research Excellence, College of Education Psychology and Social Work, Flinders University, Adelaide, Australia. ${ }^{3}$ Sleep Health Service, Repatriation General Hospital and Respiratory and Sleep Services, Southern Adelaide Local Health Network, Adelaide, Australia. ${ }^{4}$ Institute for Social Science Research, The University of Queensland, Brisbane, Australia. ${ }^{5}$ Thoracic Program, The Prince Charles Hospital, Brisbane, Australia.

Correspondence: Alexander Sweetman, Adelaide Institute for Sleep Health: a Centre of Research Excellence, Flinders University, College of Medicine and Public Health, Bedford Park, 5042, Australia. E-mail: alexander. sweetmanaflinders.edu.au

ABSTRACT Insomnia and obstructive sleep apnoea (OSA) frequently co-occur and may be causally related through sleep fragmentation and/or hyperarousal mechanisms. Previous studies suggest that OSA treatment can improve insomnia severity. However, the effect of insomnia treatment on OSA severity has not been investigated. We performed a randomised controlled trial to investigate the effect of cognitive behavioural therapy for insomnia (CBTi) on OSA severity, controlling for potential sleep-stage and posture effects.

145 patients with comorbid insomnia (International Classification of Sleep Disorders, 3rd Edn) and untreated OSA (apnoea-hypopnoea index (AHI) $\geqslant 15$ events $\cdot h^{-1}$ sleep) were randomised to a four-session CBTi programme or to a no-treatment control. Overnight sleep studies were completed pre- and posttreatment to measure AHI, arousal index and sleep architecture, to investigate the effect of intervention group, time, sleep stage (N1-3 or REM) and posture (supine or nonsupine) on OSA severity.

The CBTi group showed a 7.5 event. $\mathrm{h}^{-1}$ greater AHI difference (mean (95\% CI) decrease 5.5 (1.3-9.7) events $\cdot \mathrm{h}^{-1}$, Cohen's $d=0.2$, from 36.4 events $\cdot \mathrm{h}^{-1}$ pre-treatment) across sleep-stages and postures, compared to control (mean increase $2.0(-2.0-6.1)$ events $\cdot \mathrm{h}^{-1}, d=0.01$, from 37.5 events $\cdot \mathrm{h}^{-1}$ at pretreatment; interaction $\mathrm{p}=0.012$ ). Compared to control, the CBTi group also had a greater reduction in total number (mean difference $5.6(0.6-10.6)$ greater overall reduction; $\mathrm{p}=0.029)$ and duration of nocturnal awakenings (mean difference $21.1(2.0-40.3)$ min greater reduction; $\mathrm{p}=0.031$ ) but showed no difference in the arousal index, or sleep architecture.

CBTi consolidates sleep periods and promotes a 15\% decrease in OSA severity in patients with comorbid insomnia and OSA. This suggests that insomnia disorder may exacerbate OSA and provides further support for treating insomnia in the presence of comorbid OSA.

@ERSpublications

Cognitive behavioural therapy for insomnia consolidates sleep periods and promotes a $15 \%$ decrease in obstructive sleep apnoea severity in patients with comorbid insomnia and sleep apnoea https://bit.ly/3e $4 \mathrm{iPgB}$

Cite this article as: Sweetman A, Lack L, McEvoy RD, et al. Cognitive behavioural therapy for insomnia reduces sleep apnoea severity: a randomised controlled trial. ERJ Open Res 2020; 6: 001612020 [https://doi.org/10.1183/23120541.00161-2020].

This study is registered at https://www.anzctr.org.au/ with identifier number 12613001178730. No data from this trial will be made publicly available due to exclusion from ethics approval and participant consent.

Received: 1 April 2020 | Accepted after revision: 3 April 2020

Copyright $\odot$ ERS 2020. This article is open access and distributed under the terms of the Creative Commons Attribution Non-Commercial Licence 4.0. 


\section{Introduction}

Insomnia and obstructive sleep apnoea (OSA) are the two most common sleep disorders, each occurring in $\sim 10 \%$ of the general population $[1,2]$. OSA is characterised by repetitive brief episodes of complete (apnoea) or partial (hypopnoea) closure of the upper airway during sleep, hypoxaemia and cortical arousals. Pathophysiological mechanisms of OSA include a narrow upper airway, diminished muscle tone during sleep, unstable respiratory control (loop gain), and a low respiratory arousal threshold [3]. The most common index of the presence and severity of OSA is the apnoea-hypopnoea index (AHI), which reflects the average number of apnoea and hypopnoea events per hour of sleep. OSA patients typically have a greater AHI in "light" sleep (N1 and N2) and rapid eye movement (REM) sleep than in "deep" sleep (N3), and in supine than in nonsupine sleep [4].

Insomnia disorder is characterised by chronic difficulties initiating sleep, maintaining sleep and/or with early morning awakenings, in combination with associated daytime impairments [5]. Compared to good sleepers, insomnia patients experience increased sleep-wake transitions throughout the night, thereby increasing time spent in N1 sleep and reducing N3 sleep [6,7]. Insomnia is commonly conceptualised as a disorder of conditioned cognitive and physiological hyperarousal [8].

Comorbid insomnia and sleep apnoea (COMISA) is a common and debilitating disorder [9]. For example, $30-50 \%$ of OSA patients report insomnia symptoms, while $30-40 \%$ of insomnia patients fulfil diagnostic criteria for OSA [9-12]. These high prevalence estimates may indicate bidirectional relationships between the mechanisms and manifestations of insomnia and OSA [13-15]. One method to understand causal relationships between insomnia and OSA is to treat each disorder in isolation and measure subsequent changes in the other [16]. Although the effect of treating OSA on changes in insomnia severity has been studied $[17,18]$, the effect of insomnia treatment on changes in OSA severity in COMISA patients remains unknown.

Cognitive behavioural therapy for insomnia (CBTi) is the most effective treatment for insomnia, and has been shown to reduce the frequency of sleep-wake transitions and N1 sleep, and increase N3 sleep [19-22]. Furthermore, CBTi is effective in the presence of comorbid OSA [23-25].

As N1 sleep is associated with a higher AHI and N3 sleep is associated with lower AHI in OSA patients [4], we postulated that CBTi would reduce the overall AHI in patients with COMISA. To explore this hypothesis, we used data from a recent randomised controlled trial (RCT) in COMISA patients [25] to examine the effect of CBTi versus no CBTi on changes in objective sleep architecture, sleep parameters and respiratory events, controlling for well-established sleep stage and postural effects on AHI.

\section{Methods}

\section{Overall study design}

This substudy of a previously reported RCT was conducted to compare CBTi to a no-treatment control on changes in polysomnographic (PSG) sleep characteristics over a 6-week period in 145 COMISA patients [25]. It was hypothesised that pre- to post-treatment improvements in sleep architecture and reductions in AHI would be greater with CBTi compared to control. Given the well-established effects of sleep stage and posture on AHI [4], we included the effects of sleep stage and posture when investigating changes in respiratory events between groups and repeated measures. Hence, the effect of CBTi on respiratory outcomes was examined with a 2 (treatment group: CBTi, control) $\times 2$ (time: pre-treatment, post-treatment) $\times 2$ (posture: supine, nonsupine) $\times 4$ (stage: N1, N2, N3, REM) mixed factorial design.

Following a diagnosis of insomnia and OSA, patients were randomised to either a CBTi, or control group. Minimisation (MinimPy 0.3) incorporating biased-coin minimisation (base probability 0.7, marginal balance, 1:1 allocation ratio) was used to ensure balance in potential confounders between groups including site, AHI $\left(<30\right.$ or $\geqslant 30$ events $\left.\cdot \mathrm{h}^{-1}\right)$, sex, age $(<50$ or $\geqslant 50$ years), insomnia severity (insomnia severity index $[26]<22$ or $\geqslant 22$ ) and previous continuous positive airway pressure (CPAP) use (yes or no) [25]. The study was approved by the Southern Adelaide Clinical Human Research Ethics Committee (South Australian Local Health Network and Flinders University,), the Human Research Ethics Committee (The Prince Charles Hospital), Queensland University of Technology Human Research Ethics Committee, and the External Request Evaluation Committee (Department of Human Services, Australia).

\section{Patients}

The screening protocol, randomisation method, interventions and follow-up (figure 1) are described in detail elsewhere according to Consolidated Standards of Reporting Trials guidelines [25]. Briefly, 145 COMISA patients were recruited through sleep physicians, and online, print, television and radio advertisements at two teaching hospital sites from 2013 to 2016: the Adelaide Institute for Sleep Health, Repatriation General Hospital (South Australia) and The Prince Charles Hospital (Queensland). Patients 
were randomised by an independent pharmacy department of the Repatriation General Hospital, according to minimisation techniques to balance groups on potential confounders including insomnia severity, AHI, sex, site, prior CPAP use, and age [25].

Inclusion criteria [25] were age 18-75 years, sleep physician-diagnosed OSA according to an AHI $\geqslant 15$ events $\cdot \mathrm{h}^{-1}$ and recommendation for CPAP therapy, and psychologist-diagnosed insomnia according to International Classification of Sleep Disorders (3rd Edn) criteria [5]. Patients were excluded if they had another comorbid sleep disorder (e.g. restless leg syndrome or narcolepsy), used CPAP therapy currently or within the past 3 months, had a significant memory, perceptual or behavioural disorder, or lived too far from the research centre to conveniently attend follow-up visits.

\section{Interventions}

A description of control and CBTi content, therapist contact, adherence and treatment fidelity is presented in our previous report [25]. Briefly, a manualised CBTi programme including sleep-hygiene education, bedtime restriction therapy, sleep misperception feedback, cognitive therapy and relapse prevention was delivered by seven experienced psychologists during four 45-min sessions administered over a 6-week period.

Control patients received ongoing care from sleep physicians over a 6-week period and were offered access to CBTi following completion of the larger study [25]. Other than the four treatment sessions occurring in the CBTi group, there were no between-group differences in the number of appointments with research

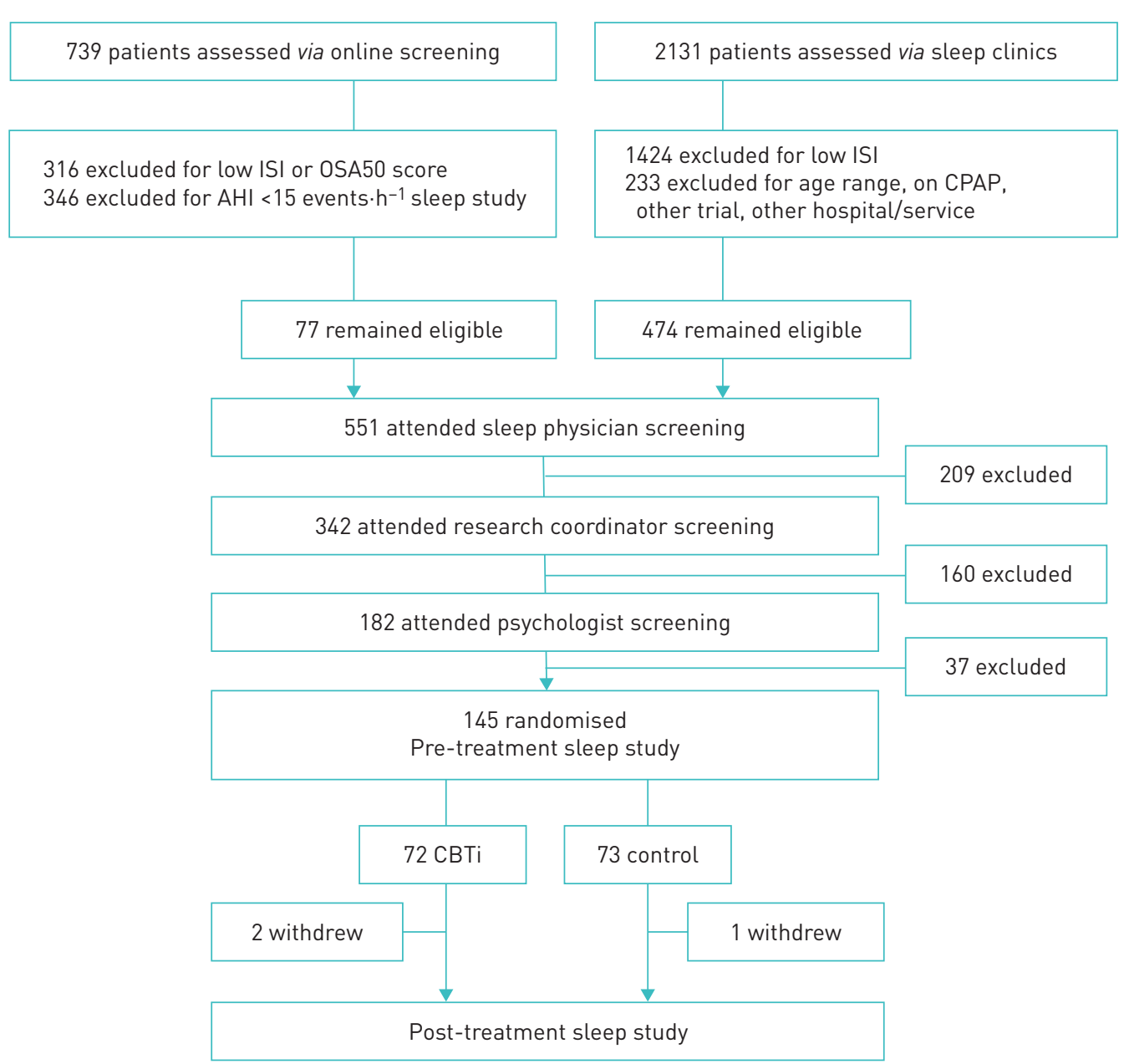

FIGURE 1 Flow diagram indicating patient screening, recruitment, randomisation and follow-up. Missing sleep study data occurred in one cognitive behavioural therapy for insomnia (CBTi) study pre-treatment, and five CBTi and two control studies post-treatment (there were no significant between-group differences in rates of missing sleep study data pre- or post-treatment; both Fisher's exact p>0.05). 71 CBTi and 73 control patients had available sleep study data for mixed model analyses. ISI: insomnia severity index; 0SA50: obstructive sleep apnoea 50 questionnaire; AHI: apnoea-hypopnoea index; CPAP: continuous positive airway pressure. 
assistants, sleep specialists or sleep technicians during treatment. All patients commenced CPAP therapy following the post-treatment PSG study. As previously reported, there were no serious adverse events related to the $\mathrm{CBTi}$ or control interventions [25].

\section{Sleep studies}

Patients completed home-based PSG sleep studies pre- and post-treatment (Somté; Compumedics, Melbourne, Australia). All recording montages included electroencephalogram (EEG) $\left(\mathrm{C}_{3}-\mathrm{M}_{2}\right.$ and $\mathrm{C}_{4}-\mathrm{M}_{1}$ ), electro-oculogram, electromyogram, ECG, pulse oximetry, nasal pressure, oronasal thermistor, chest and abdominal respiratory bands, and two leg movement channels. An accelerometer continuously indicated posture (supine, prone, left, right or upright) throughout the night, subsequently collapsed into supine and nonsupine sleep postures. Trained sleep technicians scored studies according to the American

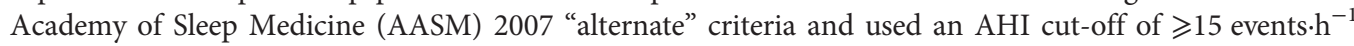
to diagnose OSA $[27,28]$. Sleep stages were categorised as N1, N2, N3 and REM sleep. Scoring technicians were blinded to the study aims and treatment allocation.

Data were extracted to determine stage- and posture-dependent average frequency of respiratory outcomes including AHI, arousal index, oxygen desaturation index (desaturations of $\geqslant 3 \%$ reduction from baseline) and average duration of respiratory events (seconds) for each PSG study. As oxygen saturation is required for the identification of hypopnoea events, any studies with oximetry drop-out $>1 \mathrm{~h}$ throughout the night were excluded from analyses of respiratory outcomes $[27,28]$. Measures of sleep architecture included total time and percentage of sleep time per stage, and total number of awakenings (defined as the total number of transitions from any "sleep" to "wake" epochs throughout the night, excluding the final awakening). Sleep parameters included total sleep time, sleep onset latency, wake after sleep onset and sleep efficiency.

\section{Statistical analyses}

Data were analysed with SPSS version 22 (IBM Statistics, Armonk, NY, USA). This substudy included all 145 patients from a larger trial, which was powered to detect a between-group difference in adherence to CPAP therapy following CBTi versus control in COMISA patients [25]. Linear mixed models including fixed effects of group (CBTi or control), time (pre- or post-treatment), posture (supine or nonsupine) and sleep stage (N1, N2, N3 or REM) were performed to examine the effect of CBTi versus control on changes in stage- and posture-dependent respiratory/arousal events from pre- to post-treatment. Mixed models including group (CBTi or control) and time (pre- or post-treatment) were used to examine the effect of CBTi versus control on changes in PSG sleep architecture and sleep parameters during treatment. Data are presented as means and 95\% confidence intervals, and p-values $<0.05$ were considered statistically significant. In the case of significant interaction or main effects, relevant pairwise post hoc comparisons were investigated using Bonferroni corrections to account for multiple contrasts. Cohen's $d$ values were calculated with pooled pre-treatment standard deviations. Following inspection of distributions for skewness (skewness $\geqslant 1$ ), logarithmic corrections were performed for oxygen desaturation index, duration of respiratory events, number of awakenings, and sleep onset latency, wake after sleep onset and sleep efficiency. As main and interaction effects of group and time remained unchanged before and after applying logarithmic corrections for normality, uncorrected outcomes are reported. Missing data were classified as missing at random and missing completely at random according to pre-defined criteria [25].

\section{Results}

\section{Pre-treatment and demographic data}

There were no significant pre-treatment differences between the CBTi and control groups in age, sex, body mass index, AHI, insomnia severity or other anthropometric measures (see previous report [25]), or objective sleep or respiratory parameters (table 1 ). Oximetry drop-out $>1 \mathrm{~h}$ during the night resulted in the exclusion of four PSG studies pre-treatment (two CBTi and two control) and four PSG studies post-treatment (three CBTi and one control) from analyses of changes in respiratory events.

\section{Sleep parameters and sleep architecture}

In addition to a previously reported greater pre- to post-treatment reduction in sleep diary- and questionnaire-based assessments of insomnia severity [25], the current analyses revealed a $21.1-\min (95 \%$ CI $19.2 \mathrm{~min}$ ) greater reduction in wake after sleep onset in the CBTi group compared to the control group (table 1).

The CBTi group also showed a $15.5-\mathrm{min}(95 \% \mathrm{CI} 14.3 \mathrm{~min})$ greater pre- to post-treatment reduction in N1 sleep duration (table 1) but with no other group by time interactions in time spent in minutes or as a percentage of sleep time in any stage (table 1). The total number of nocturnal awakenings throughout the night was also reduced from pre- to post-treatment in the CBTi but not the control group (interaction $\mathrm{p}=0.029$; CBTi group change $\mathrm{p}=0.007, d=0.2$; control group change $\mathrm{p}=0.73, d=0.0)$ (table 1 ). 
TABLE 1 Polysomnographic sleep study variables pre- and post-treatment, and between-group differences pre- and during treatment

\begin{tabular}{|c|c|c|c|c|c|c|c|c|c|}
\hline & \multicolumn{3}{|c|}{ CBTi } & \multicolumn{3}{|c|}{ Control } & \multirow[t]{2}{*}{$\begin{array}{c}\text { Pre-treatment } \\
\text { p-value }\end{array}$} & \multicolumn{2}{|c|}{$\begin{array}{l}\text { During } \\
\text { treatment }\end{array}$} \\
\hline & Pre-treatment & Post-treatment & $d$ & Pre-treatment & Post-treatment & $d$ & & $\boldsymbol{F}$ & $\mathrm{p}$-value \\
\hline Arousal index ${ }^{\#}$ events $\cdot \mathrm{h}^{-1}$ & $36.3(4.2)$ & $32.6(4.3)$ & 0.2 & $36.9(4.2)$ & $36.6(4.2)$ & 0.0 & 0.844 & 2.2 & 0.138 \\
\hline $\mathrm{ODI}^{\#}$ events$\cdot \mathrm{h}^{-1}$ & $27.1(4.8)$ & $24.3(4.9)$ & 0.1 & $30.0(4.8)$ & $30.6(4.8)$ & 0.0 & 0.954 & 0.1 & 0.224 \\
\hline $\begin{array}{l}\text { Respiratory event } \\
\text { duration }{ }^{\#} s\end{array}$ & $22.1(1.2)$ & $22.5(1.2)$ & 0.1 & $22.7(1.2)$ & $21.9(1.2)$ & 0.2 & 0.456 & 3.6 & 0.060 \\
\hline $\begin{array}{l}\text { Total number of } \\
\text { awakenings }\end{array}$ & $37.7(4.7)$ & $32.7(4.7)$ & 0.2 & $35.2(4.7)$ & $35.9(4.7)$ & 0.0 & 0.471 & 4.9 & 0.029 \\
\hline Total sleep time min & $375.2(20.5)$ & $355.4(21.0)$ & 0.2 & $358.4(20.3)$ & $363.8(20.5)$ & 0.1 & 0.305 & 2.4 & 0.123 \\
\hline Sleep onset latency min & $32.5(9.4)$ & $21.2(9.7)$ & 0.2 & $29.2(9.3)$ & $23.3(9.4)$ & 0.1 & 0.626 & 0.4 & 0.550 \\
\hline $\begin{array}{l}\text { Wake after sleep onset } \\
\text { min }\end{array}$ & $95.7(13.6)$ & $69.7(13.9)$ & 0.5 & $82.1(13.4)$ & $77.2(13.6)$ & 0.1 & 0.162 & 4.7 & 0.031 \\
\hline Sleep efficiency $\%$ & $74.3(2.9)$ & $79.3(3.0)$ & 0.4 & $75.0(2.9)$ & $76.1(2.9)$ & 0.1 & 0.747 & 2.5 & 0.115 \\
\hline N1 \% & $22.7(3.4)$ & $21.9(3.4)$ & 0.1 & $24.1(3.3)$ & $24.5(3.4)$ & 0.0 & 0.556 & 0.5 & 0.472 \\
\hline N2 \% & $48.1(2.6)$ & $47.3(2.6)$ & 0.1 & $45.7(2.6)$ & $45.0(2.6)$ & 0.1 & 0.193 & 0.0 & 0.912 \\
\hline N3 \% & $13.2(2.2)$ & $13.6(2.3)$ & 0.0 & $16.3(2.3)$ & $15.4(2.3)$ & 0.1 & 0.058 & 1.1 & 0.303 \\
\hline REM \% & $16.4(1.6)$ & $17.6(1.6)$ & 0.2 & $15.8(1.6)$ & $16.7(1.6)$ & 0.1 & 0.613 & 0.1 & 0.750 \\
\hline Supine sleep time min & $160.3(31.6)$ & $156.6(31.9)$ & 0.0 & $170.3(31.3)$ & 175.1 (31.3) & 0.0 & 0.658 & 0.3 & 0.571 \\
\hline Nonsupine sleep time min & $210.6(31.6)$ & $194.5(32.0)$ & 0.1 & $187.4(31.3)$ & $188.4(31.4)$ & 0.0 & 0.306 & 0.9 & 0.345 \\
\hline Supine sleep time $\%$ & $41.8(8.0)$ & $44.0(8.0)$ & 0.1 & $47.7(7.9)$ & $50.8(7.9)$ & 0.1 & 0.304 & 0.2 & 0.701 \\
\hline Nonsupine sleep time \% & $56.9(8.0)$ & $55.6(8.1)$ & 0.0 & $48.2(7.9)$ & $51.7(7.9)$ & 0.1 & 0.284 & 0.3 & 0.612 \\
\hline
\end{tabular}

\section{Apnoea-hypopnoea index}

There were no higher order (three- or four-way) interactions effects involving group, time, posture and/or stage, but there was a significant group by time interaction effect on AHI (figure 2 and table 1), with a 7.5 -event. $h^{-1}$ difference $(20.5 \%$ between-group change; interaction $\mathrm{p}=0.012)$ in pre- to post-treatment AHI change in the CBTi (mean (95\% CI) reduction $5.5(4.2)$ events $\cdot \mathrm{h}^{-1} ; \mathrm{p}=0.011, d=0.2$ ) compared to the control group (mean (95\% CI) increase $2.0(4.1)$ events $\cdot \mathrm{h}^{-1} ; \mathrm{p}=0.326, d=0.1$ ). Two sensitivity analyses were performed to control for pre-treatment $\mathrm{AHI}$ and insomnia severity index scores [28], respectively, which did not change the group by time interaction on AHI.

AHI was also strongly posture and stage dependent (figure 3), and was consistently greater in supine than nonsupine postures across all sleep stages $(\mathrm{p}<0.001)$, and in N1 and REM sleep compared to N2 and N3 sleep in both supine and nonsupine postures (all $\mathrm{p}<0.001)$.

\section{Arousal index, oxygen desaturation index and duration of respiratory events}

There were no three- or four-way interactions or group by time interaction effects on the arousal index, oxygen desaturation index or duration of respiratory events (table 1), but there were significant stage by posture interaction effects on arousal index (figure 3), oxygen desaturation index and duration of respiratory events.

\section{Discussion}

The main finding of the current study is that COMISA patients treated with CBTi showed a 7.5-point greater AHI difference (20.5\% between-group difference) from pre- to post-treatment across sleep stages and postures, compared to a no-treatment control group. CBTi patients also experienced a 21-min greater decrease in objective wake after sleep onset, a $15 \%$ greater reduction in the total number of nocturnal awakenings and a 15-min greater reduction in N1 sleep than control patients. These results indicate that CBTi reduces nocturnal insomnia symptoms and, to a small extent, OSA severity. This is consistent with 


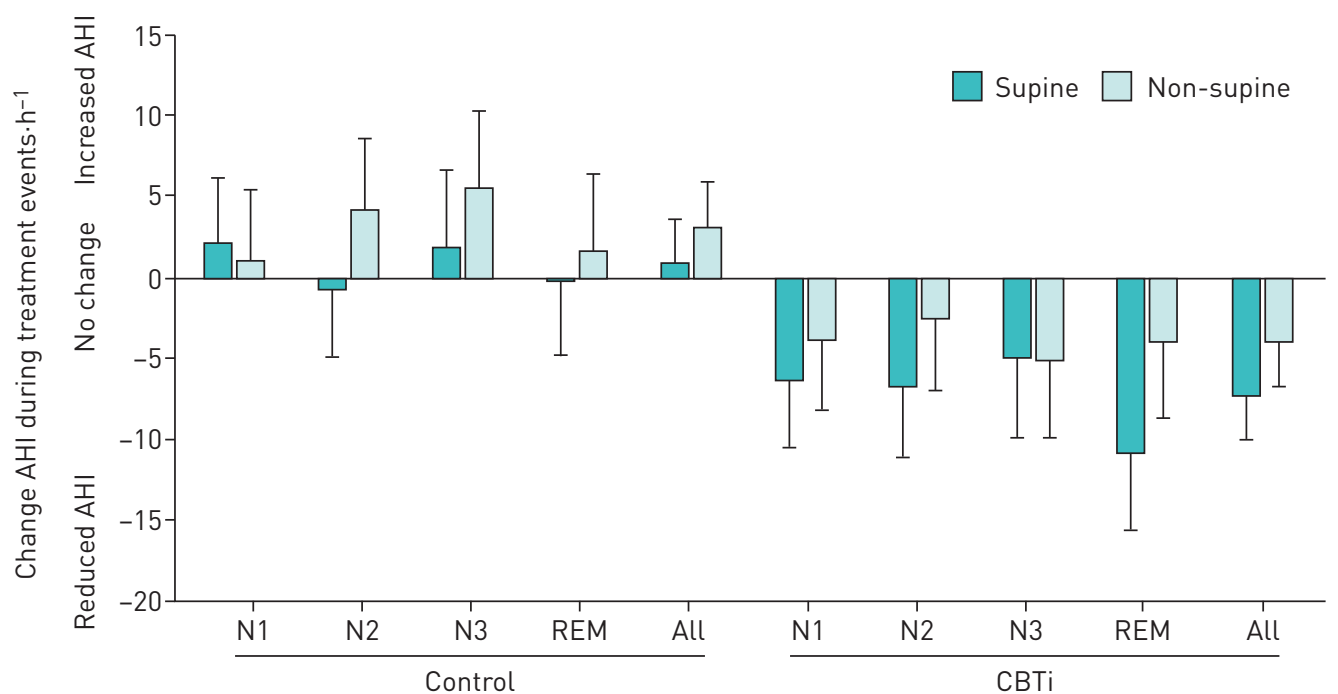

FIGURE 2 Changes in apnoea-hypopnoea index (AHI) from pre- to post-treatment between groups, sleep stage and posture. Data are presented as mean \pm SEM. CBTi: cognitive behavioural therapy for insomnia.

insomnia symptoms exacerbating OSA in COMISA patients. Given the effect of CBTi on improved acceptance and use of CPAP therapy in COMISA [25], these results provide additional support for the use of CBTi prior to commencing CPAP therapy in the $30-50 \%$ of OSA patients with comorbid insomnia symptoms $[9,11]$.

We hypothesised that CBTi would increase the percentage of sleep time in N3 sleep and reduce the percentage of sleep time in N1 sleep, thereby resulting in a decrease in the AHI $[4,20]$. Although we observed a small between-group difference in the change in N1 sleep ( $\mathrm{min}$ ) during treatment, there was no systematic change in the distribution (\%) of time spent in any sleep stage between CBTi and control groups. Furthermore, the CBTi group showed a reduction in AHI across all sleep stages, suggesting that the effect of CBTi on changes in AHI was not mediated by changes in sleep stage distribution effects.

It is possible that CBTi improves sleep continuity, which reduces manifestations of OSA in COMISA patients [16]. For example, we observed that CBTi significantly reduced the frequency and total duration of nocturnal awakenings, and time in N1 sleep, compared to the control group. Previous research has reported that partial sleep deprivation and fragmentation, and acute episodes of insomnia increase the AHI [14, 29-32]. In combination, these findings suggest that common manifestations of untreated insomnia may contribute to increased OSA severity before treatment. Hence, it is possible that CBTi

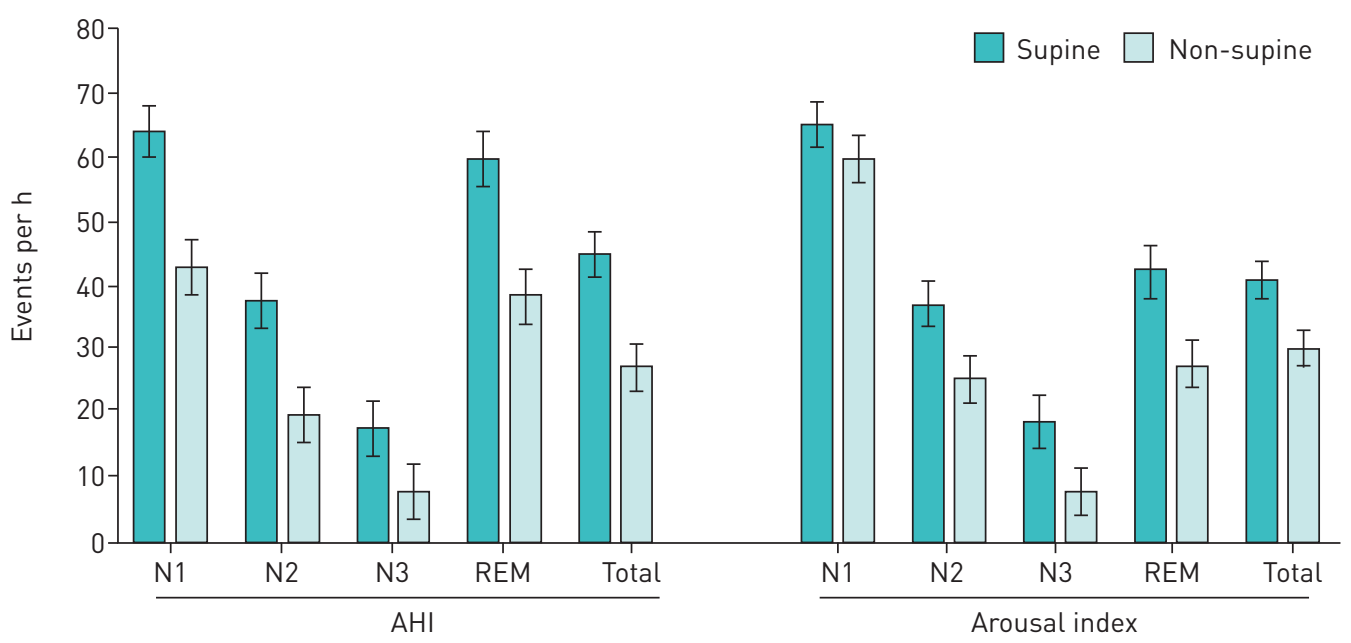

FIGURE 3 Mean apnoea-hypopnoea index (AHI) and arousal index by sleep stage and sleep posture (collapsed over intervention group and time). Data are presented as mean ( $95 \%$ confidence interval). 
consolidates periods of sleep and reduces sleep-wake transitions to improve airway stability as reflected by a small reduction in AHI.

Alternatively, it is possible that the bedtime restriction component of CBTi may increase homeostatic sleep drive throughout the night to increase the arousal threshold to both external (e.g. sound, light and temperature) and internal physiological (e.g. respiratory events) stimuli [21, 33]. Indeed, a low respiratory arousal threshold has been implicated in the pathophysiology of OSA and may be a common underlying factor in patients with COMISA [15, 34-36]. The propensity for brief arousals to transition to full awakening events may also be influenced by underlying homeostatic sleep drive. Although we did not observe an effect of CBTi on the arousal index, future research should investigate changes in the respiratory arousal threshold and relationships between cortical arousal and full awakening events in COMISA patients following CBTi $[15,34]$.

A meta-analysis of placebo-controlled studies found that among untreated OSA patients, non-benzodiazepine hypnotics do not change the AHI but are associated with a 6.6-min greater reduction in sleep onset latency and a 24-min greater reduction in wake after sleep onset [37]. In contrast, we have demonstrated that compared to a no-treatment control group, CBTi leads to a 7.5 -event $\mathrm{h}^{-1}(20.5 \%$ between-group difference) difference in AHI and a 21-min greater reduction in wake after sleep onset in COMISA patients. These improvements occurred without the risk of negative side-effects or long-term dependence associated with sedative hypnotic medications [38]. Furthermore, we recently demonstrated that CBTi decreases insomnia severity in COMISA patients, and increases subsequent acceptance and long-term use of CPAP therapy by $1 \mathrm{~h} \cdot$ night $^{-1}$ [25]. Hence, treatment of comorbid insomnia with CBTi improves insomnia symptoms, reduces the frequency of respiratory events, and increases subsequent acceptance and use of CPAP therapy in OSA patients with comorbid insomnia symptoms.

Although average sleep time appeared to show a 20-min reduction following CBTi compared to a 5-min increase following control, the interaction effect was not statistically significant. Patients who continued to apply sleep restriction techniques post-treatment could potentially reduce total sleep time through reduced time in bed. CBTi also promotes decreased wakefulness and improved perceptions of sleep (less underestimation of sleep), rather than necessarily increased objective sleep time. Furthermore, any small reduction in total sleep time immediately post-treatment often rebounds longer-term follow-up once bedtime restriction is further relaxed.

Consistent with the current findings that CBTi reduces OSA severity, the presence of bidirectional relationships is supported by previous research that demonstrates that treatment of OSA can improve specific insomnia symptoms $[17,18]$. More research is needed to understand potential bidirectional mechanistic pathways underpinning the high comorbidity of insomnia and OSA, and to develop tailored treatment approaches for different COMISA patients $[9,16]$.

\section{Limitations}

As the current study represents a substudy of data drawn from a previous RCT [25], we did not include blood sampling, high density electroencephalography or detailed physiological measures during PSG sleep studies (to measure markers of hyperarousal, respiratory arousal threshold or genioglossus activity). Future studies may consider measuring these and other physiological variables at key points during treatment to better understand the mechanisms by which insomnia and OSA interact.

Secondly, sleep stages were scored according to AASM 2007 criteria [27]. This requires the visual identification of "sleep" EEG for the majority of a 30-s epoch of sleep, and may not capture more subtle and/or more transient features of sleep disturbance in COMISA patients. Future research using more detailed and systematic EEG spectral analysis may be useful to better examine the nature of relationships between EEG disturbances and respiratory events in COMISA patients.

Finally, the current sample included adults with at least moderate insomnia (psychologist diagnosis) and OSA ( physician diagnosis, AHI $\geqslant 15$ events $\cdot \mathrm{h}^{-1}$ ), and results may not generalise to patients with different levels of insomnia and OSA severity. Therefore, future studies are required to determine the effect of CBTi on AHI in COMISA patients with mild insomnia and/or OSA.

\section{Conclusion}

Among COMISA patients, treatment of the insomnia with CBTi led to a 7.5 -event $\cdot \mathrm{h}^{-1}$ difference in AHI across sleep stages and postures, compared to a no-treatment control. CBTi also resulted in a greater reduction in the total number and duration of nocturnal awakenings and N1 sleep, but no change in other sleep/wake parameters or sleep architecture compared to control. The effect of CBTi on reduced AHI may be mediated by a reduction in nocturnal awakenings and sleep-wake transitions, or the bedtime restriction 
component of $\mathrm{CBTi}$, which may increase the respiratory arousal threshold. These data provide further support for the use of CBTi to treat insomnia symptoms in COMISA patients.

Acknowledgements: The authors would like to acknowledge the following people for their contributions to this study. Ashleigh Perry, Dr Neralie Cain, Melissa Wilson, Dr Sara Winter and Dr Lynette Buller for delivering the cognitive behavioural therapy sessions; Dr Emer Van Ryswyk, Cassandra Pattinson and Alicia Allan for managing recruitment and home studies; Tim Jarryd, Alistair Edwards, Henry Scown, Hayden Ng, Dr Gorica Micic, Dr Kalina Rossa, Dr Sherrie-Anne Kaye and Dr Luisa Roeder for assistance with sleep studies; and Laura Bandick, Michaela O’Keefe, Hiro Tojo and Carl Downey for scoring sleep studies.

Conflict of interest: A. Sweetman reports grants from the National Health and Medical Research Council, and a Collaborative Research Centre (CRC) Consortium Grant between the Australian Government, academia and industry, during the conduct of the study. L. Lack reports grants from National Health and Medical Research Council, during the conduct of the study; and grants and personal fees from Re-time Pty. Ltd. outside the submitted work. R.D. McEvoy reports grants from the National Health and Medical Research Council during the conduct of the study. S. Smith reports grants from the National Health and Medical Research Council during the conduct of the study. C.L. Chai-Coetzer reports grants from National Health and Medical Research Council during the conduct of the study. J. Douglas reports grants from National Health and Medical Research Council, during the conduct of the study. A. O'Grady reports grants from the National Health and Medical Research Council during the conduct of the study. N. Dunn reports grants from the National Health and Medical Research Council during the conduct of the study. J. Robinson reports grants from the National Health and Medical Research Council during the conduct of the study. D. Paul reports grants from the National Health and Medical Research Council during the conduct of the study, and personal fees from Philips Australia and New Zealand outside the submitted work. D. Eckert reports grants from the National Health and Medical Research Council during the conduct of the study; grants and personal fees from Bayer and Apnimed, and a Collaborative Research Centre (CRC) Consortium Grant between the Australian Government, academia and industry (industry partner: Oventus Medical), outside the submitted work. P.G. Catcheside reports grants from the National Health and Medical Research Council (NHMRC) during the conduct of the study; grants from the Australian Research Council, the Cooperative Research Centre (CRC) for Alertness, Safety and Productivity, and the NHMRC, equipment loan from Philips Respironics, and CPAP equipment provision from Air Liquide, outside the submitted work.

Support statement: This research was funded by a National Health and Medical Research Council grant (1049591; Treating insomnia comorbid with obstructive sleep apnoea: a randomised controlled clinical effectiveness trial). Funding information for this article has been deposited with the Crossref Funder Registry.

\section{References}

1 Peppard PE, Young T, Barnet JH, et al. Increased prevalence of sleep-disordered breathing in adults. Am J Epidemiol 2013; 177: 1006-1014.

2 Ohayon MM. Epidemiology of insomnia: what we know and what we still need to learn. Sleep Med Rev 2002; 6: 97-111.

3 Eckert DJ, White DP, Jordan AS, et al. Defining phenotypic causes of obstructive sleep apnea. Identification of novel therapeutic targets. Am J Respir Crit Care Med 2013; 188: 996-1004.

4 Ratnavadivel R, Chau N, Stadler D, et al. Marked reduction in obstructive sleep apnea severity in slow wave sleep. J Clin Sleep Med 2009; 5: 519-524.

5 The International Classification of Sleep Disorders. 3rd Edn. Darien, American Academy of Sleep Medicine, 2014.

6 Benca RM, Obermeyer WH, Thisted RA, et al. Sleep and psychiatric disorders: a meta-analysis. Arch Gen Psychiatry 1992; 49: 651-668.

7 Hudson JI, Pope HG, Sullivan LE, et al. Good sleep, bad sleep: a meta-analysis of polysomnographic measures in insomnia, depression, and narcolepsy. Biol Psychiatry 1992; 32: 958-975.

8 Bonnet MH, Arand DL. Hyperarousal and insomnia: State of the science. Sleep Med Rev 2010; 14: 9-15.

9 Sweetman A, Lack LC, Catcheside PG, et al. Developing a successful treatment for co-morbid insomnia and sleep apnea. Sleep Med Rev 2017; 33: 28-38.

10 Lichstein KL, Riedel BW, Lester KW, et al. Occult sleep apnea in a recruited sample of older adults with insomnia. J Consult Clin Psych 1999; 67: 405-410.

11 Krakow B, Melendrez D, Ferreira E, et al. Prevalence of insomnia symptoms in patients with sleep-disordered breathing. Chest 2001; 120: 1923-1929.

12 Sweetman A, Lack L, Bastien C. Co-morbid insomnia and sleep apnea (COMISA): prevalence, consequences, methodological considerations, and recent randomized controlled trials. Brain Sci 2019; 9: 371.

13 Guilleminault C, Eldridge FL, Dement WC. Insomnia with sleep apnea: a new syndrome. Science 1973; 181: 856-858.

14 Janssen HC, Venekamp LN, Peeters GA, et al. Management of insomnia in sleep disordered breathing. Eur Respir Rev 2019; 28: 190080.

15 Eckert DJ, Sweetman A. Impaired central control of sleep depth propensity as a common mechanism for excessive overnight wake time: Implications for sleep apnea, insomnia and beyond. J Clin Sleep Med 2020; 16: 341-343.

16 Sériès F. Can improving sleep influence sleep-disordered breathing? Drugs 2009; 69: 77-91.

17 Björnsdóttir E, Janson C, Sigurdsson JF, et al. Symptoms of insomnia among patients with obstructive sleep apnea before and after two years of positive airway pressure treatment. Sleep 2013; 36: 1901-1909.

18 Glidewell RN, Renn BN, Roby E, et al. Predictors and patterns of insomnia symptoms in OSA before and after PAP therapy. Sleep Med 2014; 15: 899-905.

19 van Straten A, van der Zweerde T, Kleiboer A, et al. Cognitive and behavioral therapies in the treatment of insomnia: a meta-analysis. Sleep Med Rev 2018; 38: 3-16.

20 Cervena K, Dauvillieres Y, Espa F, et al. Effect of cognitive behavioural therapy for insomnia on sleep architecture and sleep EEG power spectra in psychophysiological insomnia. J Sleep Res 2004; 13: 385-393. 
21 Kyle SD, Miller CB, Rogers Z, et al. Sleep restriction therapy for insomnia is associated with reduced objective total sleep time, increased daytime somnolence, and objectively-impaired vigilance: implications for the clinical management of insomnia disorder. Sleep 2014; 37: 229-237.

22 Sivertsen B, Omvik S, Pallesen S, et al. Cognitive behavioral therapy vs zopiclone for treatment of chronic primary insomnia in older adults: a randomized controlled trial. JAMA 2006; 295: 2851-2858.

23 Sweetman A, Lack LC, Lambert S, et al. Does co-morbid obstructive sleep apnea impair the effectiveness of cognitive and behavioral therapy for insomnia? Sleep Med 2017; 39: 38-46.

24 Fung CH, Martin JL, Josephson K, et al. Efficacy of cognitive behavioral therapy for insomnia in older adults with occult sleep-disordered breathing. Psychosom Med 2016; 78: 629-639.

25 Sweetman A, Lack L, Catcheside P, et al. Cognitive and behavioral therapy for insomnia increases the use of continuous positive airway pressure therapy in obstructive sleep apnea participants with co-morbid insomnia: a randomized clinical trial. Sleep 2019; 42: zsz178.

26 Bastien $\mathrm{CH}$, Vallières $\mathrm{A}$, Morin $\mathrm{CM}$. Validation of the insomnia severity index as an outcome measure for insomnia research. Sleep Med 2001; 2: 297-307.

27 Iber C, Ancoli-Israel S, Chesson A, et al. The AASM manual for the scoring of sleep and associated events: Rules, terminology and technical specification. Westchester, Ill, American Academy of Sleep Medicine, 2007.

28 Ruehland WR, Rochford PD, O'Donoghue FJ, et al. The new AASM criteria for scoring hypopneas: impact on the apnea hypopnea index. Sleep 2009; 32: 150-157.

29 Sériès F, Roy N, Marc I. Effects of sleep deprivation and sleep fragmentation on upper airway collapsibility in normal subjects. Am J Respir Crit Care Med 1994; 150: 481-485.

30 Guilleminault C. Sleep apnea syndromes: impact of sleep and sleep states. Sleep 1980; 3: 227-234.

31 Laudencka A, Klawe JJ, Tafil-Klawe M, et al. Does night-shift work induce apnea events in obstructive sleep apnea patients? J Pshysiol Pharmacol 2007; 58: 345-347.

32 Stoohs RA, Dement WC. Snoring and sleep-related breathing abnormality during partial sleep deprivation. $N$ Engl J Med 1993; 328: 1279-1279.

33 Sweetman A, McEvoy RD, Smith S, et al. The effect of cognitive and behavioral therapy for insomnia on week-to-week changes in sleepiness and sleep parameters in insomnia patients with co-morbid moderate and severe sleep apnea: A randomized controlled trial. Sleep 2020; in press [https://doi.org/10.1093/sleep/zsaa002]

34 Eckert DJ. Phenotypic approaches to obstructive sleep apnoea - new pathways for targeted therapy. Sleep Med Rev 2018; 37: 45-59.

35 Pigeon WR, Sateia MJ. Is insomnia a breathing disorder? Sleep 2012; 35: 1589-1590.

36 Younes M. Role of arousals in the pathogenesis of obstructive sleep apnea. Am J Respir Crit Care Med 2004; 169 623-633.

37 Zhang XJ, Li QY, Wang Y, et al. The effect of non-benzodiazepine hypnotics on sleep quality and severity in patients with OSA: a meta-analysis. Sleep Breath 2014; 18: 781-789.

38 Glass J, Lanctôt KL, Herrmann N, et al. Sedative hypnotics in older people with insomnia: meta-analysis of risks and benefits. BMJ 2005; 331: 1169 . 